\title{
Single Session of Low-dye Calcaneal Taping as a Viable Alternative to LASER Therapy for the Treatment of Plantar Fasciitis: A Randomized Controlled Trial
}

\author{
Shobhalakshmi S Holla ${ }^{1}$, Soni Srikantaiah ${ }^{2}$, Ramesh Debur ${ }^{3}$
}

\begin{abstract}
Background: Due to pressures of lifestyle and survival, both the urban and rural sections of society cannot give enough time for follow-up, thereby compelling many individuals to discontinue physiotherapy treatment. Treatment of plantar fasciitis (PF) requires multiple sessions and therefore falls into the scope of this problem. Taping has the advantage that it remains on the patient for multiple days and therefore negates the need for the patient's follow-up. If a single session of taping can deliver outcomes comparable to conventional LASER therapy, it would become a viable alternative solution for this problem. The aim was to compare and analyze the change in ankle muscle strength, flexibility, dorsiflexion, range of motion, and foot function in individuals with PF between the taping and LASER group.

Materials and methods: Sixty-one participants diagnosed with PF were randomly allocated into a study and control group. The study group received a single session of low-dye calcaneal taping while the control group received five sessions of LASER therapy. Both the groups received a home exercise program. A pre- and post-intervention assessment of all the variables was done for both groups.

Results: A within-group analysis using the Wilcoxon signed-rank test showed a significant difference in all the variables in both the laser and taping group $(p<0.05)$. A between-group analysis using the Mann-Whitney $U$ test showed no difference in outcomes between the taping and laser groups $(p>0.05)$.
\end{abstract}

Conclusion: A single session of taping can provide comparable outcomes as a conventional LASER therapy for short-term benefits in individuals with PF.

CTRI registration number: CTRI/2018/02/011850

Keywords: Physiotherapy, Plantar fasciitis, Plantar heel pain, Rehabilitation.

Journal of Foot and Ankle Surgery (Asia Pacific) (2022): 10.5005/jp-journals-10040-1182

\section{Background for the Study}

The current economic scenario presents unique challenges to healthcare professions, especially in developing countries. Due to pressures of lifestyle and survival, both the urban and rural sections of society cannot give enough time for follow-up. ${ }^{\text {T This }}$ challenge is much more acute in the profession of physiotherapy as many of the treatments of physiotherapy require follow-up. The cost of follow-up treatment incurred by the patients includes incidental charges such as loss of pay, travel, loss of productivity, etc. The distance traveled for follow-up physiotherapy also becomes a major concern, especially for patients residing in rural areas. Therefore, many of the patients may prefer not to continue treatment. Management of plantar fasciitis (PF) requires multiple sessions of intervention and therefore falls into the scope of this follow-up problem.

Plantar fasciitis accounts for $11-15 \%$ of the foot symptoms among adults. ${ }^{2}$ It is a degenerative process with micro-tears and fascial thickening predominating over inflammatory changes. ${ }^{3}$ The main concern in PF is heel pain during weight-bearing activities, especially walking, which is the most essential function for daily living. ${ }^{4} \mathrm{~A}$ widely accepted theory is that an increased weightbearing in the foot leads to repetitive trauma or stress that can irritate the plantar fascia at its origin on the calcaneus. ${ }^{5,6}$ The difficulty in weight-bearing and walking caused by the irritation may result in restrictions of function, social participation, and quality of life (QoL). It could also directly result in loss of muscle strength, range of motion, and muscle flexibility, thereby compromising on
${ }^{1-3}$ Department of Physiotherapy, MS Ramaiah Medical College, Bengaluru, Karnataka, India

Corresponding Author: Shobhalakshmi S Holla, Department of Physiotherapy, MS Ramaiah Medical College, Bengaluru, Karnataka, India, Phone: +91 9008025432, e-mail: pshobha76@gmail.com

How to cite this article: Holla SS, Srikantaiah S, Debur R. Single Session of Low-dye Calcaneal Taping as a Viable Alternative to LASER Therapy for the Treatment of Plantar Fasciitis: A Randomized Controlled Trial. J Foot Ankle Surg (Asia Pacific) 2022;9(1):30-34.

Source of support: Rajiv Gandhi University of Health Sciences, Bengaluru, India

Conflict of interest: None

their QoL.7 To preserve the physical function and QoL, it becomes essential to intervene through physiotherapy at the earliest.

Several non-surgical treatments have been recommended for PF which includes soft tissue mobilization, stretching, orthotics, night splints, taping, cryotherapy, phonophoresis, low-level LASER, and iontophoresis with dexamethasone. ${ }^{6}$ Kiritsi et al. investigated the effect of low-level laser therapy (LLLT) on PF through the ultrasonographic appearance of the aponeurosis and by patients' pain scores and revealed that LLLT contributes to PF healing and pain reduction. ${ }^{8,9}$

Therapeutic taping is another common clinical intervention facilitating pain reduction, joint support, proprioception, and muscle tone normalization. The 2014 clinical recommendation 
guidelines for PF suggests taping as an intervention that includes antipronation taping for immediate (up to 3 weeks) pain reduction and improved function for individuals with heel pain/ PF. $^{6}$ The low-dye calcaneal taping aims to decrease medial heel pressure by lifting the navicular bone..$^{10}$ Podolsky and Kalichman reviewed the efficacy of different taping techniques in relieving symptoms and dysfunction caused by PF. They concluded that in the short-term, taping was beneficial in treating PF and the best evidence that existed was for low-dye taping and calcaneal taping. $^{11}$

Although all treatments have reputedly provided some level of relief, the final outcome, as well as the financial and personal burden imposed by each treatment, varies markedly across protocols. Any treatment that can offer comparable outcomes using less follow-up would be a welcome change in reducing the burden of both cost incurred and time.

Taping as a method of treatment has the advantage that it remains on the patient for multiple days, thereby negating the need for the patient follow-up and also provides the necessary biomechanical correction. A single session of low-dye calcaneal taping is considered in this study as the tape is retained on the patient for 5 days and is hypothesized to produce outcomes equivalent to five sessions of LASER. Biomechanically in PF, the tape raises the medial longitudinal arch, reduces the excessive pronation of the foot, and thereby promotes healing. ${ }^{10-12}$

If a combination of a single session of taping and a home exercise program can deliver outcomes comparable to multiple sessions of a conventional therapy like LASER, it would become a viable alternative solution for this problem.

Hence, there is a need to determine if a single session of taping and home exercise program can be used as an alternative to multiple sessions of conventional LASER therapy and exercise program in subjects with PF.

The main objective of this study was to compare and analyze the change in hamstrings and tendo-Achilles flexibility, foot muscle strength, dorsiflexion ROM, and foot function between the taping and LASER group.

\section{Materials and Methods Design}

After obtaining the ethical clearance, the randomized controlled trial was conducted from February 2018 to January 2020. All patients who walked into MS Ramaiah Hospitals with PF and fulfilled the inclusion and exclusion criteria were considered for the study. Informed consent was obtained from all participants who were included in the study.

\section{Participants}

All participants were then randomly allocated into an experimental (taping) group and control (LASER) group through a computergenerated random allocation method. Participants in the age of 30-60 years who complain of medial heel pain with initial steps after a period of inactivity and worsened after prolonged weight-bearing, pain with palpation of the proximal insertion of the plantar fascia, acute to subacute pain were considered for the study. ${ }^{6}$ Subjects with fractures or other injuries to the lower limb, neurological deficits involving the lower limb, any systemic illness, inflammatory arthropathies, and usage of assistive devices were excluded from the study.

\section{Intervention}

Experimental group (Taping group): The low-dye calcaneal taping technique was used to tape the calcaneum and foot as the literature suggests it to be an effective method when compared with other forms of taping.

The taping area was first cleaned which was followed by the application of underwrap. The first strip was applied with no tension around the forefoot, proximal to the metatarsal heads. The second strip was applied with tension from just proximal to the lateral aspect of the fifth metatarsal head running proximally around the posterior aspect of the calcaneus finishing just proximal to the medial aspect of the first metatarsal head. Three to four further strips are then added similarly to the first strip with each strip overlying the more distal strip by $1 / 2$ the width of the tape. Tension is applied to these to support the arch and maintain foot position. ${ }^{10}$

\section{Calcaneal Taping}

Piece 1 was applied just distal to the lateral malleolus pulling the calcaneus medially, and was attached to the medial aspect of the foot distal to the medial malleolus. Pieces 2 and 3 follow the same pattern with an overlap of approximately one-third of the tape width moving in the distal direction. Piece 4 is put around the back of the heel starting distal to the medial malleolus. Piece 4 also serves as an anchor for the first three pieces. ${ }^{12}$ The patient was asked to retain the tape for 5 days after which they were asked to return for a follow-up assessment of outcomes.

\section{Control Group}

The control group received LLLT. The wavelength of LASER that was used for treatment was $808 \mathrm{~nm}$, with an output of $75 \mathrm{~mW}$, at $0.3 \mathrm{~J} / \mathrm{cm}^{2}$ for a duration of 7 minutes 30 seconds. The laser was given at the medial tubercle of the calcaneus which was the tender point. The group received treatment every day for 5 days following which the post-intervention assessment was done.

Both the groups received a home exercise program that consisted of stretching of tendo-Achilles, hamstrings, and plantar fascia along with strengthening of the muscles of the foot. ${ }^{13,14}$ The patients were asked to do the strengthening and flexibility exercises with a frequency of once daily with each exercise being repeated 10 times.

A detailed evaluation of the participants was done pre- and post-intervention and the following outcome measures were considered for the study.

\section{Outcome Measures}

Ankle joint range of motion was assessed using the goniometer in degrees, ankle plantar and dorsiflexor strength was measured using the handheld dynamometer in $\mathrm{Nm}^{15}$ flexibility of tendoAchilles and hamstrings was assessed using the knee to wall test ${ }^{16}$ and sit and reach test in $\mathrm{cm},{ }^{17,18}$ respectively, and function was assessed by the foot function index. ${ }^{19}$ The assessment was done by a co-investigator who was blinded to the study. All the above parameters were assessed before and after the completion of the intervention. Individuals who were on analgesics were asked to pause their medication for the duration of the study.

\section{Data Analysis}

With an alpha error of $5 \%$ and keeping the power of the study at $80 \%$, it was estimated that 32 patients to be included in each group. 
Flowchart 1: Flow of participants through the phases of the trial

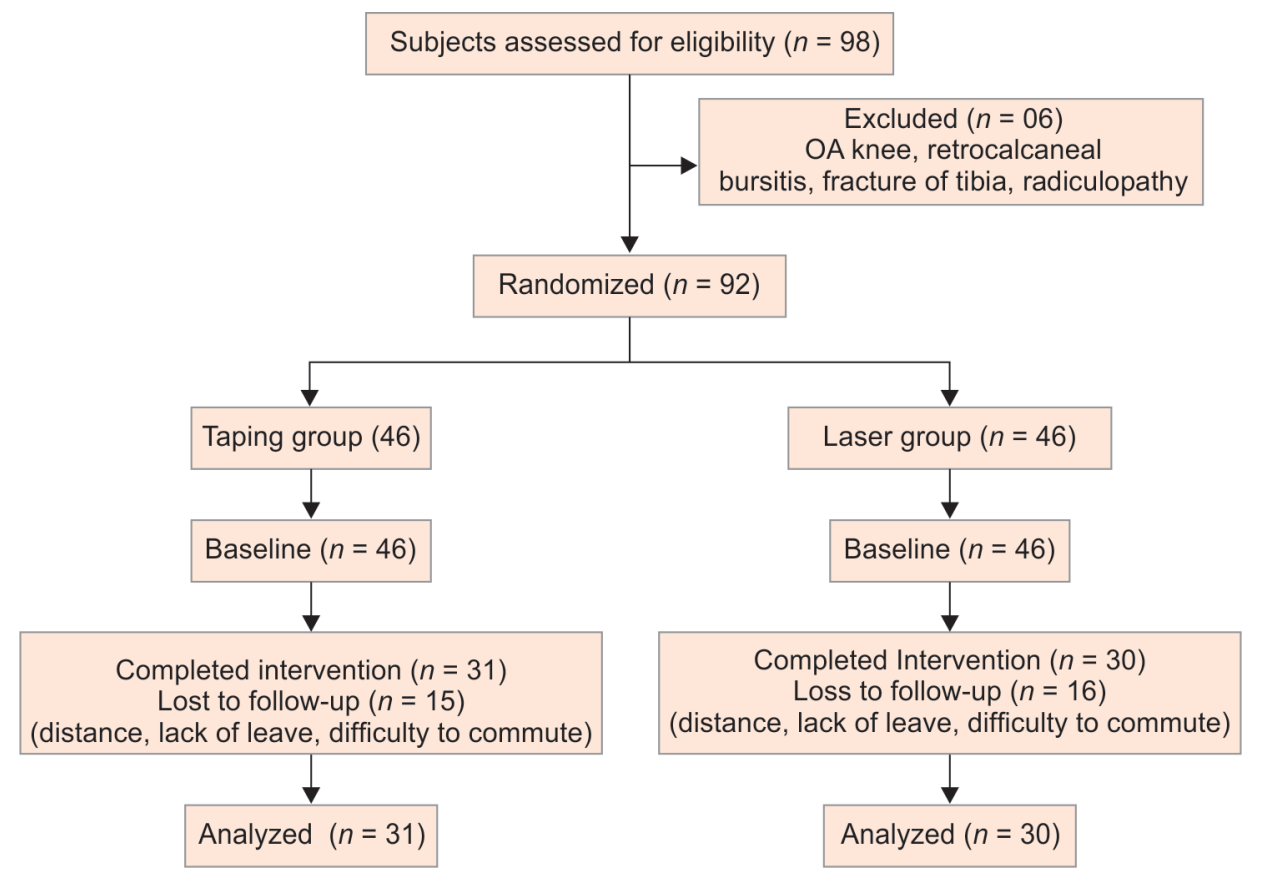

Table 1: Demographic data

\begin{tabular}{lll}
\hline & Taping group (31) & LASER group (30) \\
\hline $\begin{array}{l}\text { Age (years) } \\
\text { Gender }\end{array}$ & 42.1 & 44.7 \\
Male & 10 & 7 \\
Female & 21 & 23 \\
Side affected & Right sided-17 & Right sided-15 \\
& Left sided-14 & Left sided-15 \\
\hline
\end{tabular}

Considering dropouts, 35 was the sample size to be recruited into each group.

SPSS statistical software 23.0 was used for data analysis. Normality was assessed using Shapiro-Wilk test. As the data were not found to be normally distributed, interquartile range and nonparametric tests were used. Wilcoxon signed-rank test was used for within-group analysis and Mann-Whitney $U$ test was used for the between-group analysis of flexibility, strength, range of motion, and foot function.

Significance was kept at $p<0.05$.

\section{Results}

Ninety-eight patients who fulfilled the inclusion criteria were recruited for the study. Seven were excluded as they needed to be excluded. Ninety-one subjects were randomized into taping and LASER groups. Through the course of the study, 30 subjects did not return for follow-up. Of the 61 subjects, 31 subjects were recruited to the study group and 30 to the control group. The mean age of both the groups was 42.1 and 44.7 years, respectively, with a predominance of females in both the groups (Flowchart 1 and Table 1).

Using the Wilcoxon signed-rank test for within-group analysis, it can be seen that there is a significant difference in the hamstring and tendo-Achilles flexibility, dorsiflexion ROM, dorsiflexor, and plantar flexor muscle strength and function before and after intervention in both the taping and LASER groups $(p<0.05)$ (Table 2).
Using Mann-Whitney $U$ test for between-group analysis, it can be seen that there is no significant difference in the hamstring and tendo-Achilles flexibility, dorsiflexion range of motion, foot muscle strength, and foot function between the taping and LASER groups $(p>0.05)$ (Table 3).

\section{Discussion}

The main objective of the study was to determine if a difference in outcomes exists between a single session of taping vs multiple sessions of conventional LASER therapy in patients with PF.

On analyzing the above results, it can be seen that there is a significant difference in flexibility, dorsiflexor and plantar flexor strength, ankle dorsiflexion ROM and foot function before and after intervention in both the taping and LASER groups $(p<0.05)$.

However, a between-group analysis showed no difference in the outcomes between the taping and LASER group indicating that a single session of taping provides comparable outcomes to five sessions of LASER therapy.

Various authors have concluded that both taping and LASER therapy are beneficial in the improvement of foot function in subjects with PF. While low-dye calcaneal taping technique is found to have caused the desired beneficial effects through biomechanical corrections, LASER therapy induces analgesic effects through pain gate modulation.

Low-dye calcaneal taping raises the medial longitudinal arch of the foot and takes the force off the plantar fascia. It also inverts the heel, thereby preventing excessive pronation of the calcaneus. The tape thereby supports the arch and controls the height of the arch. ${ }^{10-12}$ This is in accordance with studies were done by Radford et al. and Hyland et al. who reported that short-term application of low-dye calcaneal taping for patients with PF was effective at reducing pain. ${ }^{10,12}$ However, in another study done by Holmes et al., it was observed that low-dye taping did not alter the foot mechanics though the pain was reduced. ${ }^{20}$

The exact mechanisms accounting for LLLT-mediated pain relief have not been identified. Some of the previous studies described 
Taping as a Viable Alternative for Plantar Fasciitis

Table 2: Comparison of flexibility, foot muscle strength, dorsiflexion ROM and foot function pre- and post-taping and LASER

\begin{tabular}{|c|c|c|c|c|c|c|}
\hline \multirow[b]{2}{*}{ Variables } & \multicolumn{3}{|c|}{ Taping group } & \multicolumn{3}{|c|}{ LASER group } \\
\hline & Pre-median (IQR) & Post-median (IQR) & $p$ value $(<0.05)$ & Pre-median (IQR) & Post-median (IQR) & $p$ value \\
\hline Hamstring flexibility & $10.0(7.0-16.0)$ & $13.0(9.0-16.5)$ & $<0.02$ & $10.0(8.5-11.8)$ & $12.0(9.25-13.5)$ & $<0.001$ \\
\hline Tendo-Achilles flexibility & $6.5(5.5-9.0)$ & $8.0(7.0-10.0)$ & $<0.03$ & $7.0(5.25-8.5)$ & $8.0(7.0-11.5)$ & $<0.001$ \\
\hline Foot function & $100(75-108)$ & $73(56-86)$ & $<0.00$ & $103(88.0-109.0)$ & $73.0(41.0-85.0)$ & $<0.001$ \\
\hline Dorsiflexion ROM & $25(20.00-27.3)$ & $29(11.00-31.3)$ & $<0.01$ & $25.00(20.00-30.00)$ & $30.00(25.00-35.00)$ & $<0.001$ \\
\hline Dorsiflexion strength & $33.4(30.8-48.7)$ & $39.4(33.4-55.7)$ & $<0.00$ & $34.4(29.8-47.7)$ & $39.3(34.8-46.0)$ & $<0.02$ \\
\hline Plantar flexor strength & $60.5(51.2-82.5)$ & $70.6(55.9-89.7)$ & $<0.01$ & $62.3(46.7-73.1)$ & $70.0(51.7-82.3)$ & $<0.02$ \\
\hline
\end{tabular}

Table 3: Comparison of flexibility, foot muscle strength, ROM, and foot function between taping group and LASER group

\begin{tabular}{llll}
\hline Variables & Mann-Whitney U & $Z$ & $p$ value \\
\hline Hamstring flexibility & 376.6 & -1.09 & $<0.29$ \\
Tendo-Achilles flexibility & 416.5 & -0.27 & $<0.79$ \\
Foot function & 405.5 & -0.45 & $<0.66$ \\
Dorsiflexion ROM & 323.0 & -1.52 & $<0.128$ \\
Dorsiflexion strength & 414.5 & -0.52 & $<0.60$ \\
Plantar flexion strength & 382.5 & -0.991 & $<0.33$ \\
\hline
\end{tabular}

a series of mechanisms that include: peripheral neural blockade, enhancement of peripheral endogenous opioids, suppression of central synaptic activity, inhibition of histamine release, modulation of neurotransmitters, promotion of adenosine triphosphate (ATP) production, reduction of muscle spasm, and increased production of anti-inflammatory cytokines. ${ }^{9,21-25}$

LASER therapy is also thought to stimulate fibrous tissue regeneration and repair processes. A lower plantar fascia thickness value and improvement in foot function was observed 6 weeks after LLLT in subjects with PF. ${ }^{8,26-28}$

The improvement in the strength of plantar flexors and flexibility of gastrocnemius and hamstrings in both the groups $(p<0.05)$ could be attributed to the home exercise program that included stretching of the plantar fascia, gastrocnemius, and hamstrings.

The improved ROM in both groups may have occurred due to the stretching exercises for the calf muscles and Achilles tendon that was given through the home exercise program. ${ }^{29}$ The strengthening exercise program of heel raises would have strengthened the plantar flexors and dorsiflexors and also contributed to the increase in tendoAchilles flexibility and dorsiflexion $\mathrm{ROM} .{ }^{14}$ A reduction in pain could have also helped in the normalization of gait, thereby indirectly strengthening the muscles of the foot. This is in accordance with a study done by DiGiovanni et al. who reported that a home exercise program is beneficial in the management of PF. ${ }^{13}$

Regarding the result on the efficacy of a single session of taping in comparison to multiple sessions of LASER therapy, it could be hypothesized that retaining the tape for a few days on the patient aids in easing off the force from the plantar fascia, thereby alleviating pain and improving foot function.

LASER therapy is an equipment-driven process that heals through fibrous regeneration and pain gate modulation and therefore needs the patient to undergo the treatment through follow-ups for a few days as a single session might not be sufficient to produce the desired effect. On reviewing the literature regarding the optimal treatment sessions of taping and LASER for short-term benefit in PF, it can be inferred that a single session of low-dye taping is retained on the patient for 3-5 days was found to be effective. ${ }^{30}$ On the contrary, lower level LASER produced the desired effect of reduced pain and improved function with 6 sessions in a span of 3 weeks. ${ }^{26}$ As there were concerns of follow-up, we had altered the number of sessions to continuous therapy for 5 days and found the beneficial effect.

On analyzing the outcomes of these two methods of treatment for PF, it can be observed that a single session of taping can be used as a viable alternative to multiple sessions of LASER therapy, thereby reducing the burden of follow-up on the patient.

\section{LIMITATIONS}

There was a minimal deficiency in the sample size as the estimated sample size could not be reached due to a heavy loss to follow-ups. The tape of a few of the subjects had to be reapplied on the 2 nd day as it came off. The patients were not asked to maintain a diary to determine the adherence to exercise.

Future studies are needed to determine the long-term efficacy of taping in comparison to multiple sessions of conventional treatment. It would be beneficial to use diagnostic imaging tools like ultrasound to determine the extent of healing.

\section{Conclusion}

It can be concluded that a single session of taping and LASER are equally effective in the short-term treatment of PF.

Since both are found to be effective it can also be concluded that a single session of low-dye calcaneal taping and home exercise program can be used as a viable short-term alternative to conventional LASER therapy and exercise program in subjects with $\mathrm{PF}$, thereby reducing the cost of treatment and burden of follow-up.

\section{References}

1. Swaminathan R, Rama R, Shanta V. Lack of active follow-up of cancer patients in Chennai, India: implications for population-based survival estimates. Bull World Health Organ 2008;86(7):509-515. DOI: 10.2471/ blt.07.046979.

2. Tahririan MA, Motififard M, Tahmasebi MN, et al. Plantar fasciitis. J Res Med Sci Off Isfahan Univ Med Sci 2012;17(8):799-804.

3. Lemont $\mathrm{H}$, Ammirati KM, Usen N. Plantar fasciitis. J Am Podiatr Med Assoc 2003;93(3):234-237. DOI: 10.7547/87507315-93-3-234.

4. Irving DB, Cook JL, Young MA, et al. Impact of chronic plantar heel pain on health-related quality of life. J Am Podiatr Med Assoc 2008;98(4):283-289. DOI: 10.7547/0980283.

5. Kwong PK, Kay D, Voner RT, et al. Plantar fasciitis. Mechanics and pathomechanics of treatment. Clin Sports Med 1988;7(1):119-126. DOI: 10.1016/S0278-5919(20)30963-7.

6. Martin RL, Davenport TE, Reischl SF, et al. Heel pain — plantar fasciitis: revision 2014. J Orthop Sports Phys Ther 2014;44(11):A1-A33. DOI: 10.2519/jospt.2014.0303. 
7. Beeson P. Plantar fasciopathy: revisiting the risk factors. Foot Ankle Surg 2014;20(3):160-165. DOI: 10.1016/j.fas.2014.03.003.

8. Kiritsi O, Tsitas K, Malliaropoulos N, et al. Ultrasonographic evaluation of plantar fasciitis after low-level laser therapy: results of a doubleblind, randomized, placebo-controlled trial. Lasers Med Sci 2010;25(2):275-281. DOI: 10.1007/s10103-009-0737-5.

9. Wang W, Jiang W, Tang C, et al. Clinical efficacy of low-level laser therapy in plantar fasciitis: a systematic review and meta-analysis. Med (Baltimore) 2019;98(3):e14088. DOI: 10.1097/MD.0000000000014088.

10. Radford JA, Landorf KB, Buchbinder R, et al. Effectiveness of low-dye taping for the short-term treatment of plantar heel pain: a randomised trial. BMC Musculoskelet Disord 2006;7(1):1. DOI: 10.1186/1471-2474-7-64.

11. Podolsky R, Kalichman L. Taping for plantar fasciitis. J Back Musculoskelet Rehabil 2015;28(1):1-6. DOI: 10.3233/BMR140485.

12. Hyland MR, Webber-Gaffney A, Cohen L, et al. Randomized controlled trial of calcaneal taping, sham taping, and plantar fascia stretching for the short-term management of plantar heel pain. J Orthop Sports Phys Ther 2006;36(6):364-371. DOI: 10.2519/jospt.2006. 2078.

13. DiGiovanni BF, Nawoczenski DA, Lintal ME, et al. Tissue-specific plantar fascia-stretching exercise enhances outcomes in patients with chronic heel pain. J Bone Jt Surg Am 2003;85(7):1270-1277. DOI: 10.2106/00004623-200307000-00013.

14. Thong-On S, Bovonsunthonchai S, Vachalathiti R, et al. Effects of strengthening and stretching exercises on the temporospatial gait parameters in patients with plantar fasciitis: a randomized controlled trial. Ann Rehabil Med 2019;43(6):662-676. DOI: 10.5535/ arm.2019.43.6.662.

15. Spink MJ, Fotoohabadi MR, Menz HB. Foot and ankle strength assessment using hand-held dynamometry: reliability and age-related differences. Gerontology 2010;56(6):525-532. DOI: 10.1159/000264655.

16. Chisholm MD, Birmingham TB, Brown J, et al. Reliability and validity of a weight-bearing measure of ankle dorsiflexion range of motion. Physiother Can 2012;64(4):347-355. DOI: 10.3138/ptc.2011-41.

17. Baltaci G, Un N, Tunay V, et al. Comparison of three different sit and reach tests for measurement of hamstring flexibility in female university students. Br J Sports Med 2003;37(1):59-61. DOI: 10.1136/ bjsm.37.1.59.

18. Mayorga-Vega D, Merino-Marban R, Viciana J. Criterion-related validity of Sit-and-Reach tests for estimating hamstring and lumbar extensibility: a meta-analysis. J Sports Sci Med 2014;13(1):1-14.
19. Budiman-Mak E, Conrad KJ, Roach KE. The foot function index: a measure of foot pain and disability. J Clin Epidemiol 1991;44(6): 561-570. DOI: 10.1016/0895-4356(91)90220-4.

20. Holmes CF, Wilcox D, Fletcher JP. Effect of a modified, low-dye medial longitudinal arch taping procedure on the subtalar joint neutral position before and after light exercise. J Orthop Sports Phys Ther 2002;32(5):194-201. DOI: 10.2519/jospt.2002.32.5.194.

21. Funk JO, Kruse $A$, Neustock $P$, et al. Helium-neon laser irradiation induces effects on cytokine production at the protein and the mRNA level. Exp Dermatol 1993;2(2):75-83. DOI: 10.1111/j.1600-0625.1993. tb00012.x

22. Hagiwara $S$, Iwasaka $H$, Hasegawa $A$, et al. Pre-irradiation of blood by gallium aluminum arsenide $(830 \mathrm{~nm})$ low-level laser enhances peripheral endogenous opioid analgesia in rats. Anesth Analg 2008;107(3):1058-1063. DOI: 10.1213/ane.0b013e31817ee43e.

23. Honmura A, Ishii A, Yanase M, et al. Analgesic effect of Ga-Al-As diode laser irradiation on hyperalgesia in carrageenin-induced inflammation. Lasers Surg Med 1993;13(4):463-469. DOI: 10.1002/ Ism.1900130411.

24. Rochkind S, Nissan M, Lubart R, et al. The in-vivo-nerve response to direct low-energy-laser irradiation. Acta Neurochir (Wien) 1988;94(12):74-77. DOI: $10.1007 / B F 01406620$.

25. Schindl A, Schindl M, Schon $H$, et al. Low-intensity laser irradiation improves skin circulation in patients with diabetic microangiopathy. Diabetes Care 1998;21(4):580-584. DOI: 10.2337/diacare.21.4.580.

26. Macias DM, Coughlin MJ, Zang K, et al. Low-level laser therapy at 635 $\mathrm{nm}$ for treatment of chronic plantar fasciitis: a placebo-controlled, randomized study. J Foot Ankle Surg 2015;54(5):768-772. DOI: 10.1053/j.jfas.2014.12.014.

27. Jastifer JR, Catena F, Doty JF, et al. Low-level laser therapy for the treatment of chronic plantar fasciitis: a prospective study. Foot Ankle Int 2014;35(6):566-571. DOI: 10.1177/1071100714523275.

28. Yinilmez Sanmak ÖD, Geler Külcü D, Mesci N, et al. Comparison of effects of low-level laser therapy and extracorporeal shock wave therapy in plantar fasciitis treatment: a randomized, prospective, single-blind clinical study. Turk J Phys Med Rehabil 2018;65(2): 184-190. DOI: 10.5606/tftrd.2019.3528.

29. Rathleff MS, Mølgaard CM, Fredberg U, et al. High-load strength training improves outcome in patients with plantar fasciitis: a randomized controlled trial with 12-month follow-up. Scand J Med Sci Sports 2015;25(3):e292. DOI: 10.1111/sms.12313.

30. Landorf KB, Radford JA, Keenan A, et al. Effectiveness of low-dye taping for the short-term management of plantar fasciitis. J Am Podiatr Med Assoc 2005;95(6):525-530. DOI: 10.7547/0950525. 